Particle Filtering for Signal Enhancement in a Noisy Shallow Ocean Environment

J. V. Candy

August 10, 2010

IEEE OCEANS 2010 Seattle, WA, United States September 18, 2010 through September 24, 2010 
This document was prepared as an account of work sponsored by an agency of the United States government. Neither the United States government nor Lawrence Livermore National Security, LLC, nor any of their employees makes any warranty, expressed or implied, or assumes any legal liability or responsibility for the accuracy, completeness, or usefulness of any information, apparatus, product, or process disclosed, or represents that its use would not infringe privately owned rights. Reference herein to any specific commercial product, process, or service by trade name, trademark, manufacturer, or otherwise does not necessarily constitute or imply its endorsement, recommendation, or favoring by the United States government or Lawrence Livermore National Security, LLC. The views and opinions of authors expressed herein do not necessarily state or reflect those of the United States government or Lawrence Livermore National Security, LLC, and shall not be used for advertising or product endorsement purposes. 


\title{
Particle Filtering for Signal Enhancement in a Noisy Shallow Ocean Environment
}

\author{
J. V. Candy, Fellow, IEEE
}

\begin{abstract}
The development of model-based processing techniques in ocean acoustics is well-known evolving from the pure statistical approach of maximum likelihood parameter estimation, matched-field processing and sequential model-based processing for Gaussian uncertainties. More recent model-based techniques such as unscented Kalman filtering $(U K F)$ and sequential Markov chain Monte Carlo $(M C M C)$ methods using particle filters $(P F)$ have been developed to improve both unimodal distribution estimates $(U K F)$ as well as multimodal estimates $(P F)$. In this paper we apply both techniques to provide enhanced signal estimates for acoustic hydrophone measurements on a vertical array and compare their performance. We use a normal-mode propagation solution to provide synthetic data in order to make the comparison and demonstrate the approach which will open the area to direct extensions such as localization, broadband processing, inversion, etc. We show how the normal-mode model can be incorporated directly into the processors along with the measurement array enabling the resulting enhancement capabilities.
\end{abstract}

\section{INTRODUCTION}

Incorporating a propagation model into a signal processing scheme has evolved over a long period of time where it was recognized that by embedding a physics-based representation can significantly improve the processing [1]-[5]. One approach, matched-field processing is based on comparing the measured pressure-field to that predicted by a propagation model to estimate source range and depth [3]-[5]. In ocean acoustics there are many problems of interest [6][11] governed by propagation models of varying degrees of sophistication. Here we are interested in a shallow water environment characterized by a normal-mode model. However, we are primarily interested in investigating the application and performance of the so-called "next generation" of modelbased signal processing algorithms, primarily the unscented Kalman filter $(U K F)$ and the particle filter $(P F)$ with the goal of analyzing their performance on synthetic data generated from a simple propagator developed using SNAP, an established normal-mode propagation model [6].

We investigate the development of a "model-based signal enhancer" that embeds a forward propagator into the processing scheme essentially mimicking previous model-based efforts that used a class of linearized processors (linearized and extended Kalman filters (LZKF, EKF)) [12], [13], [19]. In order to construct the model-based processor $(M B P)$, we first characterize the normal-mode model in terms of a statespace representation enabling a general framework for signal processing.

Model-based signal processing is concerned with the incorporation of environmental (propagation, seabed, sound speed, etc.), measurement (sensor arrays) and noise (ambient, shipping, surface, etc.) models along with measured data into a sophisticated processing algorithm capable of detecting, filtering (estimating) and localizing an acoustic source (target) in the complex ocean environment as well as adaptively estimating the model parameters themselves. These techniques are not constrained to a stationary environment which is essential in the ever changing ocean. Not only does the model-based approach offer a means of estimating various quantities of high interest, but it also provides a methodology to statistically evaluate its performance on-line [12]. Model-based techniques have been around for quite a while and have found their way into ocean acoustics [11].

Model-based processing is a direct approach that uses in-situ measurements. More specifically, the acoustic measurements are combined with a set of model parameters usually obtained from a priori information or a sophisticated simulator that solves the underlying boundary value problem to extract the initial parameters/states in order to construct the forward propagator and initialize the algorithm. The algorithm then uses the incoming data to update the parameter set jointly with the acoustic signal processing task (enhancement). In the following, we define a processor whose enhanced states are the estimated modal functions.

Background for our problem as well as the new processors is given in Section II. The design of the MBP for a shallow ocean acoustic problem is discussed in Section III and the results are given where we compare processor performance. We summarize and discuss our results in the final section.

\section{BACKGROUND}

Model-based signal processing algorithms are based on a well-defined procedure. A mathematical model of the underlying phenomenology is developed in the form of a statespace representation which allows propagation modeling and measurement uncertainties to be represented by stochastic processes. The states, in our case, the modal functions of the normal-mode model are to be estimated by a MBP. Note that if the model is assumed to be Gauss-Markov, then the resulting optimal processor is the well-known Kalman filter in the linear case [12].

For our ocean acoustic signal enhancement problem we assume a horizontally-stratified ocean of depth $h$ with a known horizontal source range $r_{s}$ and depth $z_{s}$ and that the acoustic energy from a point source can be modeled as a trapped wave governed by the Helmholtz equation. The standard separation of variables technique leads to a set of ordinary differential 
equations after separation of variables. Removing the time dependence and invoking the far-field approximation of the Hankel function, we obtain the familiar normal-mode acoustic pressure propagation model [6],

$$
p\left(r_{s}, z\right)=\sum_{m=1}^{M} q \phi_{m}\left(z_{s}\right) \phi_{m}(z) \frac{e^{-\alpha_{r}(m) r_{s}}}{\sqrt{\kappa_{r}(m) r_{s}}} e^{j \kappa_{r}(m) r_{s}}
$$

where $p$ is the acoustic pressure; $q$ is the source amplitude; $\phi_{m}$ is the $m^{t h}$ modal function; $\alpha$ is the modal attenuation; $\kappa_{r}(m)$ is the horizontal wave number associated with the $m^{t h}$ mode (as before); and $r_{s}$ is the horizontal range.

By assuming a known horizontal range a priori, we obtain a range solution given by the Hankel function, $H_{0}\left(\kappa_{r} r_{s}\right)$. Therefore, in this case, the state-space model reduces to the "depth only" [11] simplifying Eq. 1. By defining the modal coefficient

$$
\beta_{m}\left(r_{s}, z_{s}\right)=q \phi_{m}\left(z_{s}\right) \frac{e^{-\alpha_{r}(m) r_{s}}}{\sqrt{\kappa_{r}(m) r_{s}}} e^{j \kappa_{r}(m) r_{s}}
$$

then Eq. 1 becomes

$$
p\left(r_{s}, z\right)=\sum_{m=1}^{M} \beta_{m}\left(r_{s}, z_{s}\right) \phi_{m}(z)
$$

The resulting depth relation is an eigenvalue equation in $z$ with

$$
\frac{d^{2}}{d z^{2}} \phi_{m}(z)+\kappa_{z}^{2}(m) \phi_{m}(z)=0, m=1, \cdots, M
$$

whose eigensolutions $\left\{\phi_{m}(z)\right\}$ are the so called modal functions and $\kappa_{z}$ is the wave number in the z-direction. These solutions depend on the sound speed profile, $c(z)$, and the boundary conditions at the surface and bottom as well as the corresponding dispersion relation given by

$$
\kappa^{2}=\frac{\omega^{2}}{c^{2}(z)}=\kappa_{r}^{2}(m)+\kappa_{z}^{2}(m), \quad m=1, \ldots, M
$$

where $\kappa_{r}(m)$ is the $m^{t h}$ horizontal wave number in the $r$ direction and $\omega$ is the harmonic source frequency. Eq. 1 can easily be placed into state-space form, resulting in the following state equation for the $m^{\text {th }}$ mode:

$$
\frac{d}{d z} \phi_{m}(z)=\mathbf{A}_{m}(z) \phi_{m}(z)
$$

with

$$
\phi_{m}(z)=\left[\begin{array}{c}
\phi_{m}(z) \\
\frac{d}{d z} \phi_{m}(z)
\end{array}\right]=\left[\begin{array}{c}
\phi_{m 1}(z) \\
\phi_{m 2}(z)
\end{array}\right]
$$

and

$$
\mathbf{A}_{m}(z)=\left[\begin{array}{cc}
0 & 1 \\
-\kappa_{z}^{2}(m) & 0
\end{array}\right]
$$

This leads to the following $2 M$-dimensional Gauss-Markov representation of the model:

$$
\frac{d}{d z} \phi(z)=\mathbf{A}(z) \phi(z)+\mathbf{w}(z)
$$

where $\mathbf{w}(z)=\left[\begin{array}{llll}w_{1} & w_{2} & \ldots & w_{2 M}\end{array}\right]^{T}$ is additive, zero-mean Gaussian noise with corresponding covariance matrix, $\mathbf{R}_{w w}$. The system matrix $\mathbf{A}(z)$ is defined as

$$
\mathbf{A}(z)=\left[\begin{array}{ccc}
\mathbf{A}_{1}(z) & \cdots & 0 \\
\vdots & \ddots & \vdots \\
0 & \cdots & \mathbf{A}_{M}(z)
\end{array}\right]
$$

and the overall state vector is

$$
\phi(z)=\left[\begin{array}{llllll|ll}
\phi_{11} & \phi_{12} \mid \phi_{21} & \phi_{22} \mid & \ldots & \phi_{M 1} & \phi_{M 2}
\end{array}\right]^{T}
$$

This leads to the measurement equations, which we write as

$$
p\left(r_{s}, z\right)=\mathbf{C}^{T}\left(r_{s}, z_{s}\right) \phi(z)+v(z)
$$

where

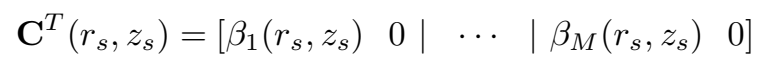

The random noise terms $\mathbf{w}(z)$ and $v(z)$ can be assumed Gaussian and zero-mean with respective covariance matrices, $\mathbf{R}_{w w}$ and $\mathbf{R}_{v v}$. The measurement noise $(v(z))$ can be used to represent the "lumped" effects of near-field acoustic noise field, flow noise on the hydrophone and electronic noise. The modal noise $(\mathbf{w}(z))$ can be used to represent the "lumped" uncertainty of sound speed errors, distant shipping noise, errors in the boundary conditions, sea state effects and ocean inhomogeneities that propagate through the ocean acoustic system dynamics (normal-mode model). These assumptions result in a Gauss-Markov model with optimal solution to the state estimation problem a Kalman filter [13].

Since our array spatially samples the pressure-field, we choose to discretize the differential state equations using a central difference approach for improved numerical stability, that is, from Eq. 4 we have

$$
\frac{d^{2}}{d z^{2}} \phi_{m} \approx \frac{\phi_{m}\left(z_{\ell}\right)-2 \phi_{m}\left(z_{\ell-1}\right)+\phi_{m}\left(z_{\ell-2}\right)}{\triangle z_{\ell}^{2}}
$$

for $\triangle z_{\ell}:=z_{\ell}-z_{\ell-1}$. Applying this approximation to Eq. 4 gives

$\phi_{m}\left(z_{\ell}\right)-2 \phi_{m}\left(z_{\ell-1}\right)+\phi_{m}\left(z_{\ell-2}\right)+\triangle z_{\ell}^{2} \kappa_{z}^{2}(m) \phi_{m}\left(z_{\ell-1}\right)=0$

where $z_{\ell}$ is the location of the $\ell$-th sensor. Defining the discrete modal state vector as $\phi_{m}\left(z_{\ell}\right):=\left[\phi_{m}\left(z_{\ell-2}\right) \mid \phi_{m}\left(z_{\ell-1}\right)\right]^{T}$, we obtain the discrete form of our state-space model given by Eq. 6 with the $A$-submatrices defined by

$\mathbf{A}_{m}(z):=\left[\begin{array}{cc}0 & 1 \\ -1 & 2-\triangle z_{\ell}^{2} \kappa_{z}^{2}(m)\end{array}\right] ; \quad m=1, \cdots, M$ 


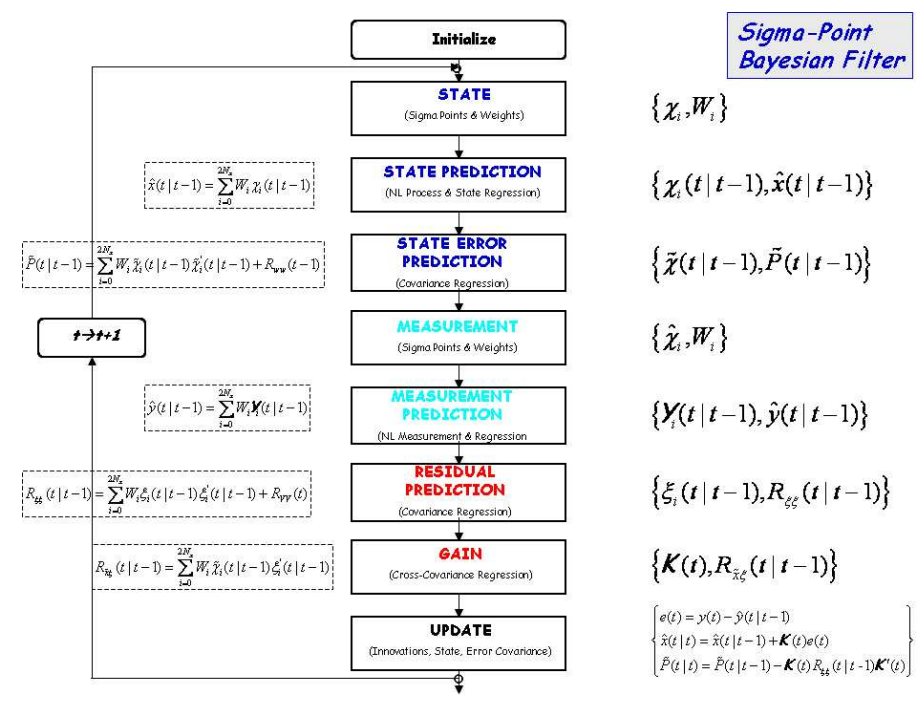

Fig. 1. Unscented Kalman filter algorithm flow diagram: initialization, prediction, update and innovation with $t$ the index variable.

Before we attempt to describe the "new approaches" to the estimation problem, let us put these techniques in perspective. The $U K F$ is an alternative to the nonlinear or extended Kalman filter processor applied successfully in many of the modelbased ocean acoustic applications [1]-[11]. Like the EKF it is still restricted to a unimodal distribution (single peak), but that distribution need not be Gaussian. It also performs a linearization (statistical), but not of the system dynamical model, but of an inherent nonlinear vector transformation requiring "sigma points" which deterministically characterize the underlying unimodal distribution. These points have been pre-calculated for the Gaussian case [13]. It has been shown that the $U K F$ clearly outperforms the $E K F$ and its variants (iterated $E K F$, higher order EKFs, etc.). and is more accurate and precise besides being much easier to implement, since Jacobian are no longer required.

A detailed flow diagram of the $U K F$ is shown in Fig. 1 where we note the basic predictor/update structure. Much of the algorithm is devoted to the statistical linearization in which regression estimators are used to perform the transformation while the usual Kalman filtering equations are used to perform the updates. We refer the interested reader to the current texts or basic papers for more details [14]-[19].

A particle filter, on the other hand, is a completely different approach to nonlinear filtering in that it removes the restriction of additive Gaussian noise sources and is clearly capable of characterizing multimodal distributions. In fact, it might be easier to think of the $P F$ as a histogram or kernel density like estimator in the sense that it is an empirical probability mass function $(P M F)$ that approximates the desired posterior distribution such that statistical inferences can easily be performed and statistics extracted directly. Here the idea is a radical change in thinking where we attempt to develop an empirical estimation of the posterior distribution following a purely Bayesian approach using Monte Carlo (MC) sampling theory as its enabling foundation. As one might expect the computational burden of the $P F$ is much higher that of $\mathrm{KF}$, since it must provide an estimate of the underlying state posterior distribution component-by-component at each $z_{\ell^{-}}$ step along with the fact that the number of samples to characterize the distribution is equal to the number of particles.

$$
\hat{p}\left[\phi\left(z_{\ell}\right) \mid P_{z}\right]=\sum_{i=1}^{N_{p}} \hat{W}_{i}\left(z_{\ell}\right) \delta\left(\phi\left(z_{\ell}\right)-\phi_{i}\left(z_{\ell}\right)\right) \quad \forall z
$$

$$
\begin{array}{cl}
\hat{W}_{i}\left(z_{\ell}\right) & \propto \hat{\operatorname{Pr}}\left[\phi_{i}\left(z_{\ell}\right)\right] \text { is the estimated weights at depth } z_{\ell} ; \\
\phi_{i}\left(z_{\ell}\right) & \text { is the } i \text {-th particle at depth } z_{\ell} ; \\
\hat{p}[\cdot] & \text { is the estimated empirical distribution; } \\
P_{z} & \text { is the set of batch pressure-field measurements, } \\
& P_{z}=\left\{p\left(z_{1}\right) \cdots p\left(z_{L}\right)\right\} .
\end{array}
$$

So we see that once the underlying posterior is available, the estimates of important statistics can be extracted directly. For instance, the maximum a posteriori (MAP) estimate is simply found by locating the location of the particular particle $\hat{x}_{i}\left(z_{\ell}\right)$ corresponding to the maximum of the $P M F$, that is

$$
\hat{\Phi}_{i}^{M A P}(z)=\max _{i} \quad \hat{p}\left[\phi_{i}(z) \mid P_{z}\right]
$$

A detailed flow diagram of the particle filter (bootstrap) algorithm is shown in Fig. 2 illustrating the prediction and update steps along with a resampling algorithm to provide convergence. Again more details can be found in the referenced textbooks and papers [14]-[19]. Note also that if we place the EKF/UKF into this framework, then we see that the underlying posterior distribution has already been decided to be multivariate Gaussian with the objective to extract the corresponding conditional mean and covariance as accurately as possible. Therefore, we see that the UKF provide the multivariate posterior solution

$$
\begin{gathered}
\hat{p}\left[\phi\left(z_{\ell}\right) \mid P_{z}\right]=(2 \pi)^{N_{x} / 2}\left|R_{\phi \phi}\left(z_{\ell} \mid z_{\ell}\right)\right|^{-1 / 2} \\
\exp \left\{-\frac{1}{2}\left(\phi\left(z_{\ell}\right)-\hat{\phi}\left(z_{\ell} \mid z_{\ell}\right)\right)^{T} R_{\phi \phi}^{-1}\left(z_{\ell} \mid z_{\ell}\right)\left(\phi\left(z_{\ell}\right)-\hat{\phi}\left(z_{\ell} \mid z_{\ell}\right)\right)\right\}
\end{gathered}
$$

where $\hat{\phi}\left(z_{\ell} \mid z_{\ell}\right)$ is the conditional modal mean at depth $z_{\ell}$ and $R_{\phi \phi}\left(z_{\ell} \mid z_{\ell}\right)$ is the conditional modal covariance based on pressure-field measurements up to depth $z_{\ell}$.

So we see that there exists a fundamental philosophical difference between the UKF (Kalman) processor and the PF processor. Their implementations are completely different as well: one based on approximating the required distribution through statistical linearization and one through an empirical PMF estimator.

\section{MODEL-BASED OCEAN ACOUSTIC PROCESSING}

In this section we develop the basic processors and apply them to the normal-mode signal enhancement problem defined more formally in terms of our state-space representations as: 


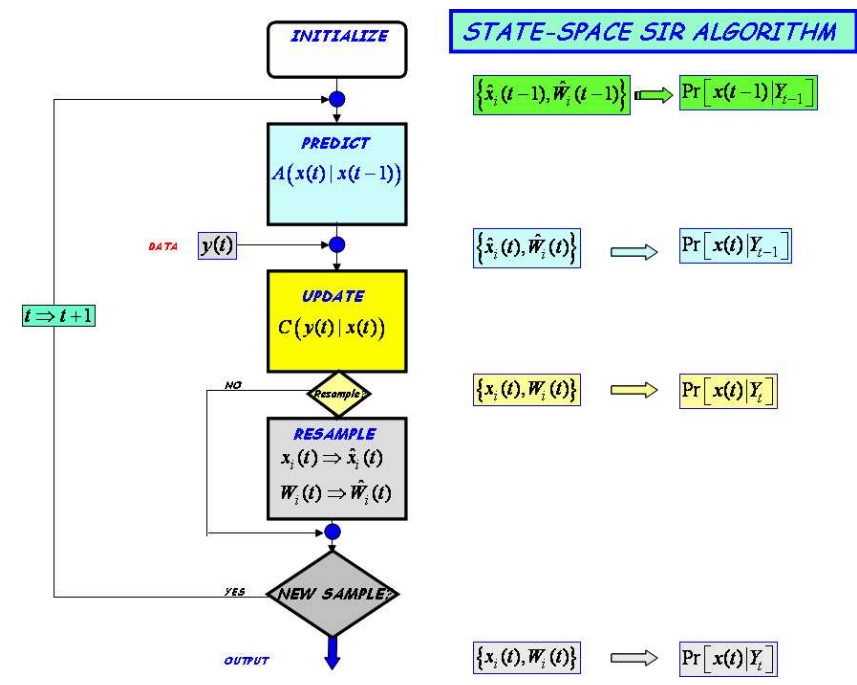

Fig. 2. Bootstrap particle filter algorithm flow diagram: prediction, update and resampling with $t$ the index variable.

GIVEN a set of noisy pressure-field and sound speed measurements, $\left[\left\{p\left(r_{s}, z_{\ell}\right)\right\},\left\{c\left(z_{\ell}\right)\right\}\right]$ and the underlying state-space model of Eqs. 6 and 12, FIND the "best" (minimum error variance) estimate of the modal functions, that is, $\left\{\hat{\phi}_{m}(z \mid z)\right\} ; m=$ $1, \cdots, M$ and measurements (enhanced) $\left\{\hat{p}\left(r_{s}, z_{\ell}\right)\right\}$.

\section{A. Simulation}

We synthesized data using the SNAP normal-mode propagation model assume a shallow ocean environment of $100 \mathrm{~m}$ depth with a unity amplitude, $100 \mathrm{~Hz}$ point source located at $25 \mathrm{~m}$ and a range of $10 \mathrm{~km}$. The sound speed profile followed a summer pattern [6]. Here a 100 -element vertical array is deployed from the bottom with $1.0 \mathrm{~m}$ separation spanning the entire water column and clearly over-sampling to avoid any potential artifacts in synthesizing the pressure-field. The SNAP solution supported 4 modes with corresponding average horizontal wave numbers: $\{0.417,0.413,0.407,0.400\} m^{-1}$.

\section{B. Results}

We performed a series of "tuning" runs for both the UKF and $P F$. We primarily adjusted the process noise covariance matrix for each of the modal functions and then executed an 100 member ensemble of realizations using these parameters. The particle filter was designed with the same parameters and 500 particles were used to characterize the posterior PMF at each depth. Resampling was applied at every iteration of the $P F$ [19] to avoid any potential degradation. The resulting figures show the averaged MAP PF estimates (in thick red lines) while the UKF are in the dotted turquoise line. In Fig. 3, we see the enhanced (predicted) pressure-field estimates (thick red line) along with the UKF estimates and raw data. It is clear from this figure that both estimators are capable of tracking and enhancing the pressure-field. Using classical performance metrics, the zero-mean whiteness tests, both processors satisfy the criteria of unbiasedness (zero-mean: $2.9 \times 10^{-10}<0.25$ )

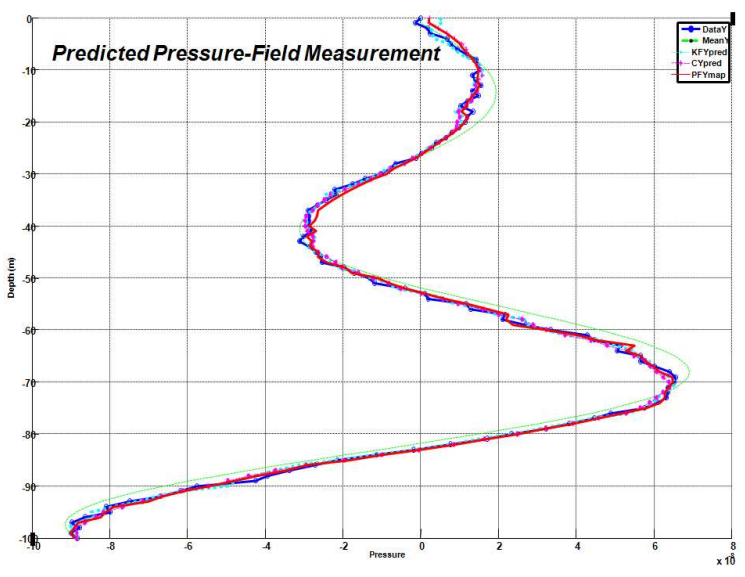

Fig. 3. PF and UKF Pressure-Field Estimation: Raw Data, UKF, PF MAP.

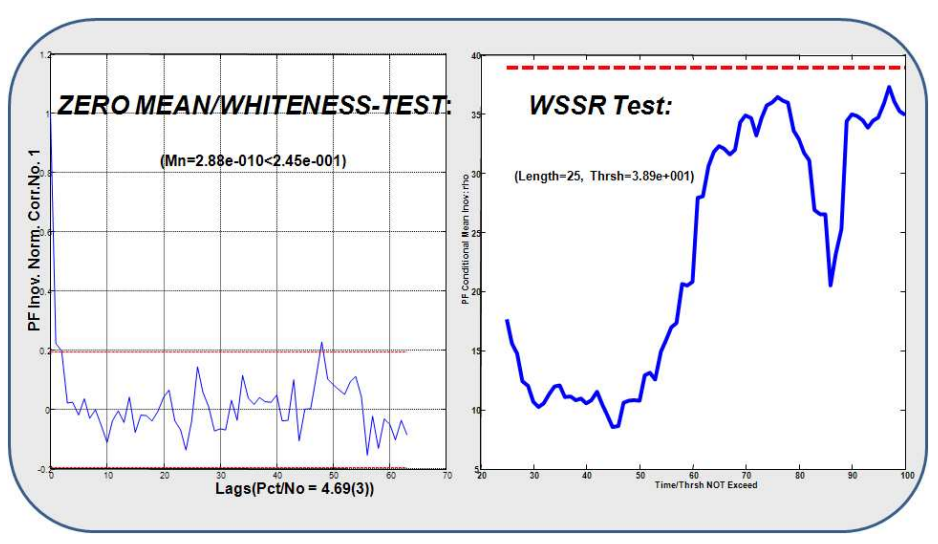

Fig. 4. PF and UKF Pressure-Field Estimation: Zero-Mean/Whiteness and WSSR statistical tests.

and uncorrelated residuals (innovations), that is, less than $5 \%$ exceeding the bound $(4.7 \%)$ as illustrated in Fig. 4. The weighted sum-squared residual (WSSR) test is also applied with satisfactory results, that is, no samples exceed the threshold (see [13] for details).

The modal estimates for both the $U K F$ and $P F$ are shown in Fig. 5 along with the raw data (blue circles). It appears from the figure that the $P F$ tracks slightly better than the $U K F$ over the ensemble. The allocation of the particles are shown in Fig. 6 where we observe the four modal PMFs along exhibiting multimodal estimates and just how the particles are allocated to the highest probability regions. That explains why the $P F$ performs slightly better. Further analysis of the complete modal PMFs indicate the multimodal evolution of the posterior $P M F$ and the enhanced pressure-field $P M F$ are shown in Fig. 7-9.

\section{SUMMARY}

In this paper we have developed on-line model-based solutions to the ocean acoustic signal processing problem based on the normal-mode propagation model and a vertical sensor array measurement system. The algorithms employed were the unscented Kalman filter and the particle filter both modern 

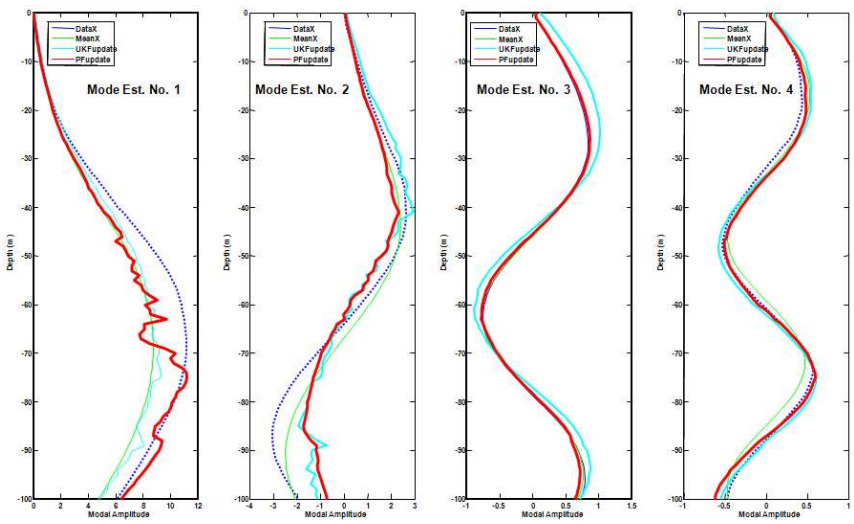

Fig. 5. PF and UKF Modal (State) Estimation (Modes 1-4): Raw Data, UKF, $P F$ Conditional Mean, $P F$ MAP.

Normal-Mode PMF Estimates at $\mathrm{z}=75 \mathrm{~m}$ (Modes 1-4)
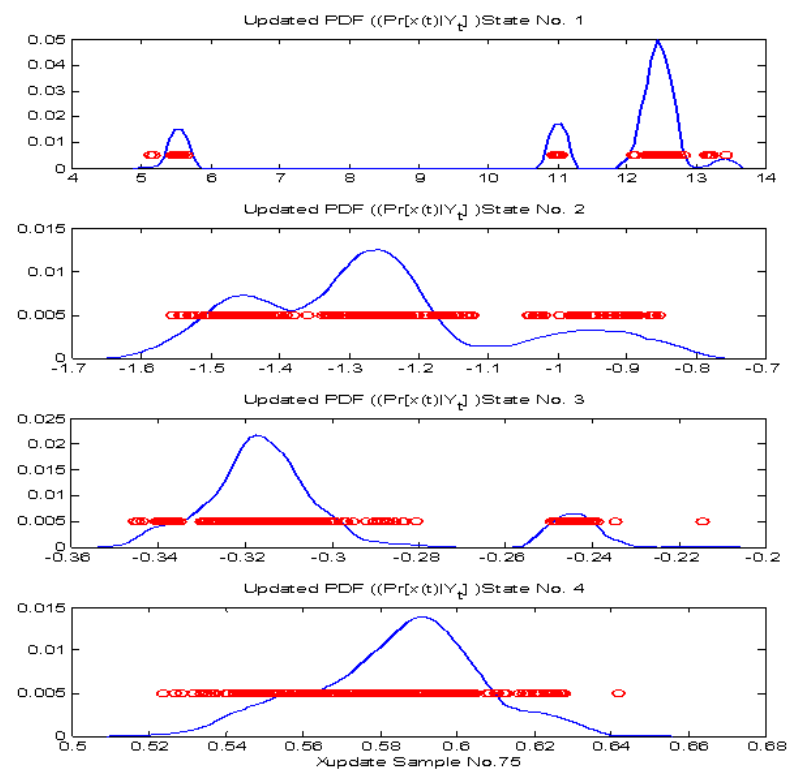

Fig. 6. PF Modal (State) PMFs (Modes 1-4): Particle grouping (coalesense).

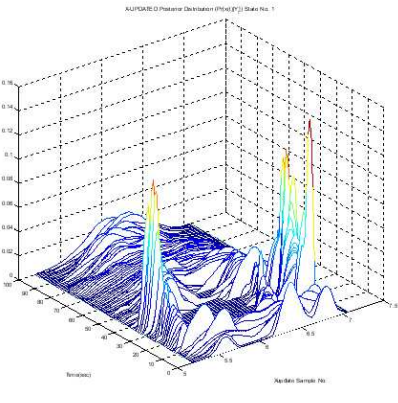

(a) MODE No. 1 PMF

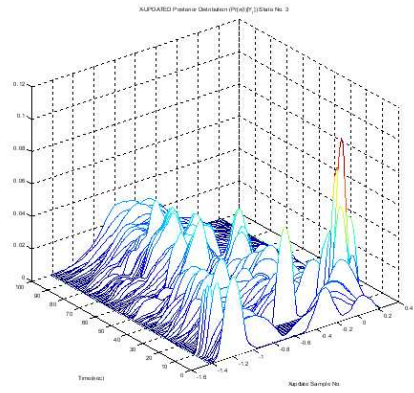

(b) MODE No. 2 PMF
Fig. 7. PF and UKF Modal (State) PMF Estimation (Modes 1-2).

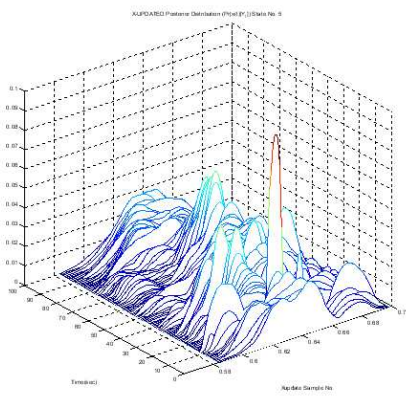

(a) MODE No. 3 PMF

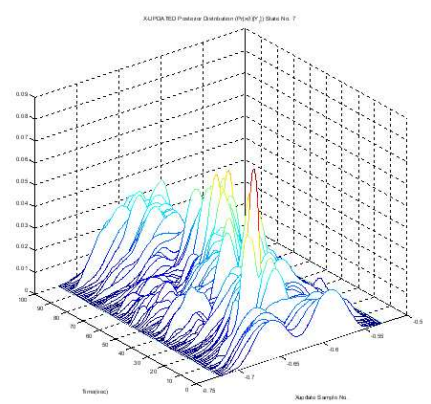

(b) MODE No. 4 PMF
Fig. 8. PF and UKF Modal (State) PMF Estimation (Modes 3-4).

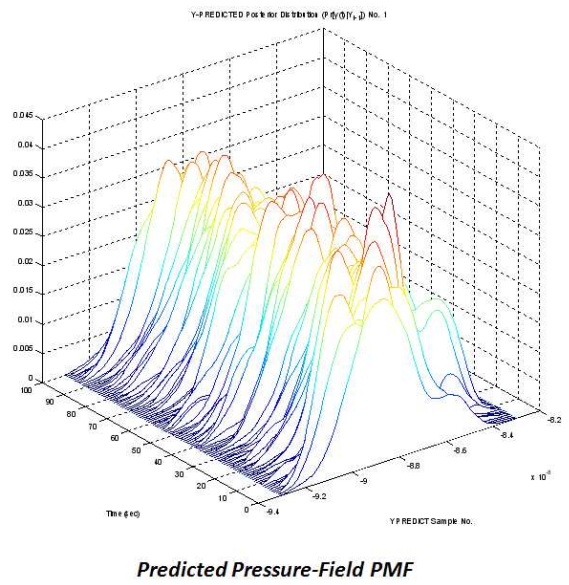

Fig. 9. PF Pressure-Field PMF Estimation (particle vs. time vs. probability.

approaches to apply to this problem. We compared their performances and found slightly better performance of the $P F$ over a 100-member ensemble. Much more effort must be applied to gain a full understanding of applying these approaches to usual ocean acoustic problems (localization, tracking, inversion, etc.). Our future efforts will be focused on extending the processors to those problems.

\section{ACKNOWLEDGEMENTS}

This work performed under the auspices of the U.S. Department of Energy by Lawrence Livermore National Laboratory under Contract DE-AC52-07NA27344.

\section{REFERENCES}

[1] M. J. Hinich, "Maximum likelihood signal processing for a vertical array," J. Acoust. Soc. Am., 54, 499-503, 1973.

[2] C. S. Clay, "Use of arrays for acoustic transmission in a noisy ocean,' Res. Geophys., 4, (4), 475-507, 1966.

[3] H. P. Bucker, "Use of calculated sound fields and matched-field detection to locate sound in shallow water," J. Acoust. Soc. Am., 59, 329-337, 1976.

[4] A. M. Richardson, and L. W. Note, "A posteriori probability source localization in an uncertain sound speed, deep ocean environment," $J$. Acoust. Soc. Am., 89, (6), 2280-2284, 1991.

[5] E. J. Sullivan and D. Middleton, "Estimation and detection issues in matched-field processing", IEEE J. Oceanic Eng., 18, (3), 156-167, 1993. 
[6] F. B. Jensen and M. C. Ferla, "SNAP: the SACLANTCEN normal-model propagation model," Report SM-121, Italy: SACLANTCEN, 1979.

[7] M. B. Porter, "The KRACKEN normal mode program," Report SM-245, Italy: SACLANTCEN, 1991.

[8] H. Schmidt, "SAFARI: Seismo-acoustic fast field algorithm for range independent environments," Report SM-245, Italy: SACLANTCEN, 1987.

[9] F. B. Jensen, Kuperman, W. A., Porter M. B.,and H. Schmidt, Computational Ocean Acoustics New York: Amer. Instit. Physics Press, 1994.

[10] J. V. Candy and E.J. Sullivan. "Model-based processor design for a shallow water ocean acoustic experiment", J. Acoust. Soc. Am., 95, (4) 2038-2051, 1994.

[11] J. V. Candy and E. J. Sullivan. "Ocean acoustic signal processing: a model-based approach." J. Acoust. Soc. Am., 92, (12), 3185-3201, 1992

[12] A. Jazwinski, Stochastic Processes and Filtering Theory. New York:Academic Press, 1970.

[13] J. V. Candy, Model-Based Signal Processing. Hoboken, N.J.:Wiley/IEEE Press, 2006. New York:McGraw-Hill, 1986.

[14] B. Ristic, S. Arulampalam and N. Gordon, Beyond the Kalman Filter: Particle Filters for Tracking Applications, Boston: Artech House, 2004.

[15] O. Cappe, E. Moulines and T. Ryden, Inference in Hidden Markov Models, New York: Springer-Verlag, 2005.

[16] S. Godsill and P. Djuric, "Special Issue: Monte Carlo methods for statistical signal processing." IEEE Trans. Signal Proc., vol. 50, 2002.

[17] P. Djuric, J. Kotecha, J. Zhang, Y. Huang, T. Ghirmai, M. Bugallo and J. Miguez, "Particle Filtering," IEEE Signal Proc. Mag. vol. 20, No. 5, 19-38, 2003.

[18] S. Haykin and N. de Freitas, "Special Issue: Sequential state estimation: from Kalman filters to particle filters." Proc. IEEE, vol. 92, No. 3, 399574, 2004.

[19] J. V. Candy, Bayesian Signal Processing: Classical, Modern and Particle Filtering Methods. Hoboken, N.J.:Wiley/IEEE Press, 2009. 